\title{
Engineering Semiconductor Quantum Dots for Cellular, Molecular, and In Vivo Imaging
}

\author{
A.M. Smith and S.M. Nie* \\ * Departments of Biomedical Engineering and Chemistry, Emory University and Georgia Institute \\ of Technology, Atlanta, GA 30342.
}

Semiconductor quantum dots are fluorescent nanoparticles with unique optical and electronic properties that have attracted interest across a broad range of disciplines, from bioimaging to solar energy conversion. The 'mesoscopic' size of these $\sim 2-10 \mathrm{~nm}$ particles spans the threshold between small molecules and large crystals, resulting in size-tunable fluorescence and absorption. For bioimaging applications, these particles offer many useful properties that cannot be attained from traditional organic dyes and fluorescent proteins, such as broadband excitation, continuously tunable fluorescence with narrow emission bands, efficient near-infrared emission, and a tremendous resistance to photodegradation. Despite recent use of these particles in a wide variety of bioimaging applications, many challenges still remain in the development, implementation, and understanding of these new probes. Most importantly, conventional quantum dots contain heavy metal atoms that may result in acute cellular toxicity, and the hydrodynamic size these particles is too large for many applications requiring compact probes.

We have determined that a promising means of reducing the heavy metal toxicity of semiconductor quantum dots is through lattice strain [1]. A limiting physical phenomenon for quantum dots is that semiconductor materials generally only have visible and near-infrared emission if they contain heavy atoms (e.g. cadmium or lead). However, the absorption and fluorescence may be vastly tuned by straining the crystal lattice, through compression or tension. We have found that such strain may be imposed through overgrowth of a compressive or expansive shell material, which modifies the bond length of both the core material and shell material. With the use of a small and soft core material (CdTe), overgrowth of a $\mathrm{ZnSe}$ shell, containing no heavy metal atoms, not only allows the reduction of heavy metal composition by up to 20 -fold compared to traditional nanocrystals, but it also allows dynamic control over the wavelength $(500-1000 \mathrm{~nm})$, the excited state lifetime, location of charge carriers, and the overlap between the fluorescence and absorption spectra. We have studied the effect of materials composition of these particles with regard to their cellular toxicity in great depth [2]. Surprisingly, we found that the cytotoxicity of these nanocrystals is only marginally related to their heavy metal composition, and that surface reactions on the colloids dominate their effect on cellular growth and function. This finding demonstrates that the traditional understanding of cytotoxicity must be significantly modified to accommodate the unique attributes of nanomaterials.

The interactions between nanoparticles and biological components, such as biomolecules and plasma membranes, are largely dependent on the size and the surface properties of the particles. Smaller nanocrystals not only diffuse faster and are less affected by steric hindrance than larger nanocrystals, but they have also been found to escape systemic circulation via kidney excretion [3] and extravasation [4], and their intracellular location can be significantly altered [5]. However the amphiphilic polymers that are commonly used to coat the surfaces of colloidal quantum dots are too bulky to yield compact hydrodynamic sizes, resulting particles with 20-30 nm diameter. Small ligands may be alternatively used to stabilize quantum dots but their bond with the surface is too 
unstable to prevent deterioration of the particles in biological media. To this end, we have prepared a new coating technology based on multidentate polymer ligands [6]. An important finding is that a linear polymer containing a balanced composition of thiol and amine coordinating groups can coat nanocrystals with a compact hydrodynamic size and exceptional colloidal stability. This has resulted in a new generation of brightly fluorescent quantum dots with hydrodynamic sizes between 5.6 to $9.7 \mathrm{~nm}$ with tunable fluorescence from the visible to the near-infrared. These new particles have enabled us to find that there are specific size thresholds for many interactions between quantum dots and biological macromolecules, suggesting that minimizing the size of the nanoparticle probe is crucial for maximizing the utility of these biolabeling agents.

\section{References}

[1] A.M. Smith, A.M. Mohs and S.M. Nie, Nat. Nanotechnol., 4 (2009) 56.

[2] A.M. Mohs, A.M. Smith, M.C. Mancini, and S.M. Nie, manuscript submitted.

[3] H.S. Choi, W. Liu, P. Misra, E. Tanaka, J.P. Zimmer, B.I. Ipe, M.G. Bawendi, J.V. Frangioni, Nat. Biotechnol., 25 (2007) 1165.

[4] J.P. Zimmer, S.W. Kim, S. Ohnishi, E. Tanaka, J.V. Frangioni, M.G. Bawendi, J. Am. Chem. Soc., 128 (2006) 2526.

[5] I. Nabiev, S. Mitchell, A. Davies, Y. Williams, D. Kelleher, R. Moore, Y.K. Gun'ko, S. Byrne, Y.P. Rakovich, J.F. Donegan, A. Sukhanova, J. Conroy, D. Cottell, N. Gaponik, A. Rogach, Y. Volkov, Nano Lett. 7 (2007) 3452.

[6] A.M. Smith and S.M. Nie, J. Am. Chem. Soc., 130 (2008) 11278.

[7] This work was supported by grants from the National Institutes of Health (P20 GM072069, R01 CA108468, and U01HL080711, U54CA119338). 